Proceedings of the Nineteenth Annual IEEE Semiconductor Thermal Measurement and Management Symposium, March 11-13, 2003, San Jose, CA, Institute of Electrical and Electronics Engineers, Inc.

For more information contact G.Recktenwald: gerry@me.pdx.edu

\title{
Optimization of a Phase Change Heat Sink for Extreme Environments
}

\author{
Jason Leland and Gerald Recktenwald \\ Mechanical Engineering Department \\ Portland State University, \\ Portland, Oregon 97207-0751
}

\begin{abstract}
Results of numerical optimization are reported for a phase change heat sink used to cool electronic equipment in extreme environments. The heat sink consists of a conventional, extruded aluminum sink embedded in a block of phase change material. This type of heat sink is used in infrared cameras carried by fire fighters into burning buildings.

Optimization of the geometry of the heat sink assembly involves determination of the fin length, the fin thickness, and the base thickness that maximizes the time time before the base of the heat sink reaches a critical temperature. The numerical model is briefly described, and representative results of the simulation are presented. The optimum design for a given combination of heat load, conductance to ambient, and phase change material is discussed. The model can easily be applied to other geometries, heat loads, and material properties.
\end{abstract}

\section{INTRODUCTION}

Phase change materials (PCMs) can be used to absorb excess heat during peaks in transient power dissipation by electronics. If the peaks are intermittent and of short duration, a PCM can reduce the size of the cooling system (saving money, space, or other system resources) by allowing the system to temporarily function above its continuous cooling capacity.

Pal and Joshi [1] analyzed and conducted experiments on a phase change heat sink for avionics applications. O'Connor and Weber [2] measured the performance of a phase change heat sink. Wirtz and his students [3], [4], [5] used numerical models and experiments to optimize the performance of phase change heat sinks. Fosset et. al [6] used a microencapsulated PCM for an aircraft communications device. Hodes et. al [7] report on the use of PCM to extend operation of a communications hand set.

Phase change materials have also been used in a variety of energy storage applications. For example, Brousseau and Lacroix [8] used a numerical model similar to ours to predict the behavior of a device for load-levelling in an electrical power grid.

Here we consider a different application of a phase change heat sink: as the primary heat reservoir for electronics operating in an extreme environment. Specifically, we analyze a heat sink for an infrared imaging systems are used by fire fighters to navigate through smoke-filled buildings. Inside a burning building, the ambient air can be well above $85^{\circ} \mathrm{C}$, a common temperature limit for electronics, so rejection of heat to the ambient is not possible. In fact, for successful operation, the electronics must be insulated from the ambient.

In this application, the phase change heat sink provides a temporary low temperature heat reservoir which is used to cool the electronics. The goal of optimizing the heat sink is to extend the time of useful operation of the heat sink of a given volume.

\section{Heat Sink Design}

Figure 1 is a schematic of the phase change heat sink, which consists of a conventional, extruded aluminum heat sink embedded in a phase change material (PCM). The base of the heat sink is attached to a CPU, or to a heat spreader from the CPU and other electronics. The PCM is enclosed in an insulated housing. A protective case separates this assembly from the ambient.

A simple volumetric analysis shows that the aluminum makes less contribution to the heat storage capacity than the PCM. Consider the heat storage capacity of the device in Figure 1. Assume that the assembly experiences a uniform temperature change $\Delta T$ that spans the melting temperature $T_{m}$ of the PCM. The heat stored in the aluminum fin is $E_{f}=\rho_{f} c_{f} V_{f} \Delta T$, where $\rho_{f}$ and $c_{f}$ are the density and heat capacity of the fin, and $V_{f}$ is the volume occupied by the fin. The heat stored in the PCM is $E=\rho V(c \Delta T+\lambda)$, where $\rho$ and $c$ are the average density and heat capacity of the PCM, $V$ is the volume occupied by the PCM, and $\lambda$ is the latent heat of fusion of the PCM. Let $V_{\text {tot }}=V_{f}+V$ and $E_{\text {tot }}=E_{f}+E$, then

$$
\frac{E_{\text {tot }} / V_{\text {tot }}}{\rho c \Delta T}=r+(1-r+\text { Ste }) \frac{V}{V_{\text {tot }}}
$$

where $r=\rho_{f} c_{f} /(\rho c)$ and Ste $=\lambda /(c \Delta T)$ is the Stefan number. Since $r$ and Ste are always positive, and for most PCMs, $r<1$, Equation (1) shows that the heat storage capacity of the assembly is always increased by increasing the volume fraction of PCM.

The key assumption in the preceding volumetric analysis is that the temperature of the assembly is uniform. This condition will not prevail during the temperature transient because the thermal diffusivity of the PCM is typically much less than the thermal diffusivity of the fin. Although the metallic fin reduces the ultimate heat storage capacity, the fin enhances the heat transport from the edge of the assembly to its core. Optimizing the transient performance of the heat sink involves finding the spatial distribution of the aluminum that provides a high rate of heat transfer at the base without exceeding a threshold temperature of the base.

\section{A. Simplified Model}

Figure 2 is a diagram of the two-dimensional model used to optimize the heat sink. A single repeating module of width $w$ is chosen to represent the entire assembly. Symmetry conditions imposed on the left and right boundaries $(x=0$ and $x=w)$ neglect the effect of heat gain from the ambient through the insulated edges on the left and right in Figure 1. The symmetry 


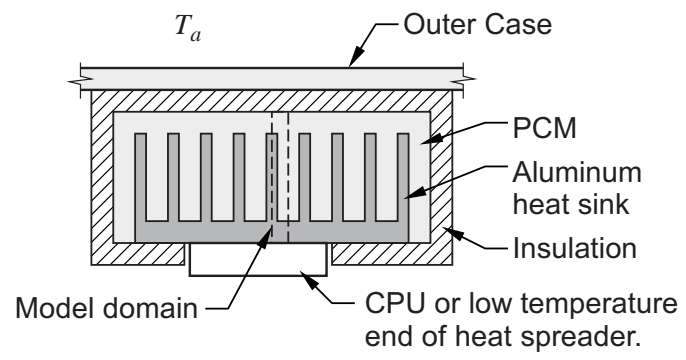

Fig. 1. Phase change heat sink for extreme environments. The heat sink is insulated to minimize heat gain from the surroundings.

conditions also neglect the heat spreading resistance due to the finite size of the heat source.

The lack of proper edge effects and head spreading in the base, limit the generality of the results presented in this paper. Despite the limitations of the model, it provides insight into the relationship between heat conduction in the metal fin and heat conduction with phase change in the PCM.

The top surface of the PCM is connected to the ambient via a conductance boundary condition

$$
q(x)=U_{a}\left(T-T_{a}\right)
$$

where $q$ is the local heat flux, $U_{a}$ is an overall conductance, $T$ is the temperature of the PCM on the boundary of the domain, and $T_{a}$ is the ambient temperature. The conductance value is obtained from a one-dimensional model of the thermal resistance of the insulation layer, the outer case, and the heat transfer coefficient on the surface of the outer case. For all calculations reported here, $U_{a}=7220 \mathrm{~W} / \mathrm{m}^{2} /{ }^{\circ} \mathrm{C}$.

Our model also neglects the possibility of buoyancy induced flow. Motion of the liquid PCM would enhance the heat transfer by increasing the rate at which heat is transported across the molten layer. This is not likely to be a large effect, however. For the honeycomb geometry investigated by Pal and Joshi [1], free convection did not make a significant contribution to the heat transfer for Rayleigh numbers as high as $9.1 \times 10^{6}$. With $\Delta T=80-47=33^{\circ} \mathrm{C}, w=5 \mathrm{~mm}, \beta=1.1 \times 10^{-3} \mathrm{~K}^{-1}$ (see [9]), and the material properties from Table I,

$$
\mathrm{Ra}=\frac{g \beta \Delta T(2 w)^{3}}{\nu \alpha} \sim 3 \times 10^{5}
$$

This is significantly above the commonly used critical Rayleigh number of 1000 used to indicate the onset of free convection. (See, e.g. [10]). The Ra is also significantly below the range of $\mathrm{Ra}$ investigated by Pal and Joshi.

Without explicit verification of the importance of free convection (e.g., by including it in our model), we cannot unequivocally say that free convection is unimportant. As a practical matter, a heat sink design that was strongly influenced by (internal) free convection would be less predictable in the field. Motion in the liquid PCM would make the performance of the heat sink sensitive to orientation and acceleration (shock). Other practical PCM heat sink designs avoid this problem by using dry PCMs [4], [5], microencapsulated PCMs [6], or PCMs embedded in expanded metal foam [3], [6].

Another idealization of our model is that the densities of the solid and liquid phases of the PCM are equal. This is not true for

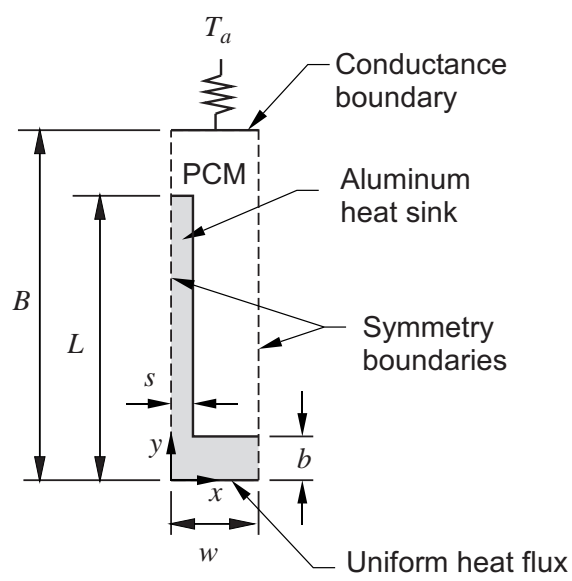

Fig. 2. Two-dimensional computational domain representing a repeating module of the heat sink. The module is identified by the dashed line in Figure 1

paraffin. Although density changes in the material introduces practical design challenges, it is unlikely to affect the predicted thermal performance of the model.

\section{NUMERICAL MODEL}

Transient conduction in the solid material (Aluminum) is coupled with the transient conduction and phase change in the PCM. Analytical solutions exist for melting in uniform semiinfinite solids. For the geometry under consideration here, detailed analysis of the transient heat transfer requires a numerical model.

\section{A. Governing Equations}

In the absence of convective motion in the liquid phase, the energy equation for a material undergoing solid/liquid phase change is

$$
\frac{\partial H}{\partial t}=\nabla \cdot(k \nabla T)
$$

where $H$ is the enthalpy per unit volume, $k$ is the effective thermal conductivity, and $T$ is the temperature. The phase of the material is quantified by the local liquid fraction, $g$ where $0 \leq g \leq 1: g=1$ indicates the fully liquid state, and $g=0$ indicates the fully solid state. Intermediate values of $g$ correspond to "mushy" states in materials that do not exhibit a sharp phase change. For the results presented here, we only consider a sharp phase change where the liquid/solid transformation occurs at a fixed temperature $T_{m}$.

In the numerical model, $g$ is considered to be uniform throughout a control volume. Values of $g$ between zero and one are possible, and correspond to a melt front somewhere within the control volume. For example, $g=0.25$ (in the model) for a material undergoing isothermal phase change indicates that three quarters of the cell is solid and one quarter of the cell is liquid.

The enthalpy accounts for the local composition (liq$\mathrm{uid} / \mathrm{solid} /$ mushy) as well as the latent heat. Ignoring the possibility of a change in volume during the phase change, the enthalpy per unit volume is

$$
H=\rho(1-g) \int_{T_{\mathrm{ref}}}^{T} c_{s} d \xi+\rho g \int_{T_{\mathrm{ref}}}^{T} c_{\ell} d \xi+\rho g \lambda
$$


where $\rho$ is the material density (assumed to be the same for liquid and solid states), $T_{\text {ref }}$ is a reference temperature used to set $H=0, c_{s}$ and $c_{\ell}$ are the specific heats of the solid and liquid, respectively, and $\lambda$ is the latent heat of fusion.

If $c_{s}$ and $c_{\ell}$ are independent of $T$, and if $T_{\text {ref }}=0$, then Equation (4) simplifies to

$$
H=\rho\left[\left((1-g) c_{s}+g c_{\ell}\right) T+g \lambda\right] .
$$

Differentiating Equation (5) with respect to time, and substituting the result into Equation (3) and rearranging gives

$$
\rho c \frac{\partial T}{\partial t}=\nabla \cdot(k \nabla T)-\rho\left[\left(c_{\ell}-c_{s}\right) T+\lambda\right] \frac{\partial g}{\partial t}
$$

where $c$ is the mixture specific heat

$$
c=(1-g) c_{s}+g c_{\ell} .
$$

Equation (6) is in the form of the transient heat conduction equation with the source term $-\rho\left[\left(c_{\ell}-c_{s}\right) T+\lambda\right] \frac{\partial g}{\partial t}$.

\section{B. Implementation}

Numerical solution of Equation (6) is obtained with a relatively straightforward adaptation of a transient heat conduction code. We use a fixed grid, control-volume finite-difference model of transient heat transfer in a material undergoing a solidto-liquid phase change. Motion (convection) of the liquid phase is ignored.

The details of the phase change model are all contained in the source term, and correct linearization of this term is critical. The method of Voller and Swaminathan [11] is used linearize the source term and to update the liquid fraction at each time step. Following Voller and Swaminathan, we use a fully-implicit time integration scheme. Additional details are in [12].

The model was implemented in MATLAB. Our original plan was to use MATLAB to prototype the code, and then translate it to $\mathrm{C}$ for better computational efficiency. Typical execution times for the fine mesh runs were around two hours on a 2.2 GHz Pentium 4 computer. For our purposes the run times were acceptable, and the conversion to $\mathrm{C}$ was deferred.

The code was validated by comparing the numerical solutions it produced to the analytical solution for melting of a subcooled, semi-infinite solid that experiences a step changes in surface temperature. See [13] for the analytical solution and [12] for details of the validation runs.

\section{Optimization Strategy}

The geometry was optimized to maximize $\tau^{*}$, the time at which the heat sink base achieved a temperature of $80^{\circ} \mathrm{C}$. The value of $\tau^{*}$ represents the amount of time that the device will be functional when running in an environment with extreme ambient conditions. Maximizing $\tau^{*}$ results in an electronic device that can operate longer under adverse conditions. Alternatively, for a given $\tau^{*}$, one could minimize the weight by decreasing the size of the heat sink and PCM assembly.

A second indicator of performance is $f^{*}$, the melt fraction when $t=\tau^{*}$. If $f^{*}<1$, the PCM is not fully melted when the base temperature exceeds the threshold temperature. Thus, a good heat sink design will have large $\tau^{*}$ and $f^{*}$ close to one.
TABLE I

FIXED MODEL PARAMETERS.

\begin{tabular}{lll}
\hline Aluminum & $\rho$ & $2707 \mathrm{~kg} / \mathrm{m}^{3}$ \\
& $k$ & $204 \mathrm{~W} / \mathrm{m} /{ }^{\circ} \mathrm{C}$ \\
& $c$ & $896 \mathrm{~J} / \mathrm{kg} / \mathrm{K}$ \\
\hline PCM & $\rho$ & $818 \mathrm{~kg} / \mathrm{m}^{3}$ \\
& $k_{s}, k_{\ell}$ & $0.240 \mathrm{~W} / \mathrm{m} /{ }^{\circ} \mathrm{C}$ \\
& $c_{s}$ & $2950 \mathrm{~J} / \mathrm{kg} / \mathrm{K}$ \\
& $c_{\ell}$ & $2510 \mathrm{~J} / \mathrm{kg} / \mathrm{K}$ \\
& $\lambda$ & $2.66 \times 10^{5} \mathrm{~J} / \mathrm{kg}$ \\
& $T_{m}$ & $47^{\circ} \mathrm{C}$ \\
\hline Dimensions & $w$ & $5 \mathrm{~mm}$ \\
\hline Thermal BC and $\mathrm{IC}$ & $T_{a}$ & $80^{\circ} \mathrm{C}$ \\
& $T_{0}$ & $25^{\circ} \mathrm{C}$ \\
& $U_{a}$ & $7220 \mathrm{~W} / \mathrm{m}^{2} /{ }^{\circ} \mathrm{C}$ \\
& $q_{b}$ & $16,000 \mathrm{~W} / \mathrm{m}^{2}$ \\
\hline
\end{tabular}

\section{A. Fixed Model Parameters}

Table I lists the model parameters that were held constant during the optimization. The PCM is Suntech P116 with the property values given by Brousseau and Lacroix [8].

\section{B. Manual Search for Optimum}

Optimization of the heat sink involved determining the combination of $L, s$ and $b$ for a given heat input $q_{b}$, module width $w$, ambient boundary condition $\left(T_{a}\right.$ and $\left.U_{a}\right)$, and given PCM properties. In addition, the optimum $L$ was determined from three values of $B$, the overall module height.

The search for the optimum combination of $L, s$, and $b$ was performed manually. Values of $L, s$, and $b$ were varied one at a time. While $L$ was varied, $s$ and $b$ were held fixed. The a value of $L$ that maximized $\tau^{*}, s$ was varied next, while $L$ and $b$ were fixed. Finally, $b$ was varied while $L$ and $s$ were held fixed. A second series of adjustments to $L, s$, and $b$ was performed to find a better estimate of the global optimum.

The manual search process was sufficient for our purposes. An automated search method, such as that employed by Zheng and Wirtz [4], [5], would be desirable if a larger set of parameters (or alternative inputs for $q_{b}, w, T_{a}, U_{a}$, and PCM properties) were to be considered.

\section{Coarse and Fine Mesh Solutions}

The search for the optimum was performed with a coarse and a fine mesh. For each parameter $(L, s$, and $b)$ a coarse mesh with typically $16 \times 40$ control volumes was used to maximize $\tau^{*}$. A series of runs on a finer mesh, typically $40 \times 100$, was used to confirm the results obtained with the coarse mesh. The time steps (of the model) for the coarse and fine mesh solutions were 0.15 and 0.02 seconds, respectively. In all cases the optimums obtained with the coarse and fine meshes were in very good agreement.

\section{RESULTS}

Before discussing the results of optimizing the heat sink performance, we show some typical transient behavior of the heat 
TABLE II

MOdel PARAMETERS FOR TRANSIENT RESUltS PRESENTED IN FiguRE 3 AND FIGURE 4.

\begin{tabular}{cccc}
\hline & Case A & Case B & Optimum \\
\hline$L / B$ & 0.85 & 0.90 & 0.888 \\
$b / B$ & 0.15 & 0.05 & 0.012 \\
$s / w$ & 0.15 & 0.20 & 0.275 \\
\hline
\end{tabular}
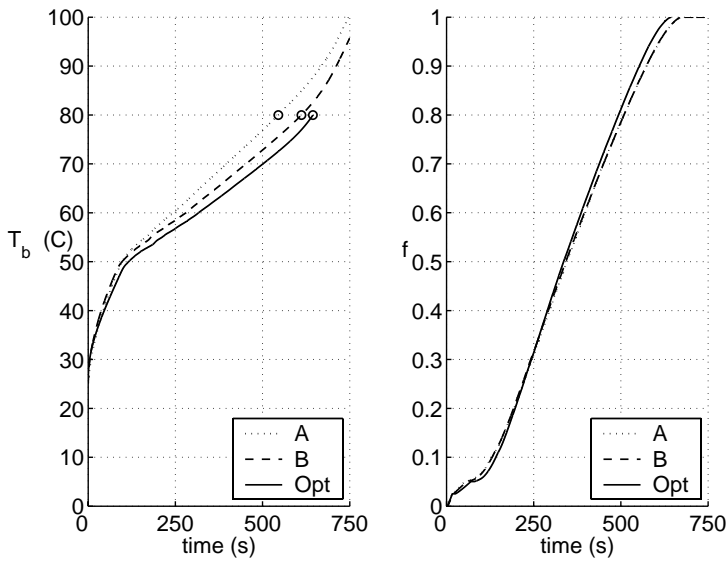

Fig. 3. Variation of base temperature and melt fraction. Parameter values for Case A, Case B, and the optimum solution are defined in Table II.

sink. This helps explain the mechanisms that affect the thermal performance of the heat sink.

\section{A. Typical Transient Behavior}

Table II lists the geometric parameters for three of the cases studied during the heat sink optimization. Case $\mathrm{A}$ is an arbitrarily chosen reference geometry. Case B represents a heat sink with better performance than Case A. The optimum geometry (third column of Table II) is the result of the final optimization discussed later in this paper.

Figure 3 shows the variation of base temperature and melt fraction for the three configurations of the heat sink specified in Table II. The base temperature is defined as the area-weighted temperature of the heated surface at the bottom of Figure 2.

At $t=0$ the entire domain is at $25^{\circ} \mathrm{C}$. Heat input at the base causes the base temperature to rise. All three cases show similar $T_{b}(t)$ curves until $T_{b} \approx 50^{\circ} \mathrm{C}$, which is near the melting temperature $T_{m}=47^{\circ} \mathrm{C}$. Note, as indicated in the right half of Figure 3, that the PCM begins melting at the beginning of the simulation, i.e. before the base temperature rises above the melting temperature. The melting first occurs at the top boundary due to heat gain from the ambient.

In the middle section of the simulation, the $T_{b}(t)$ curves diverge for the three models. Case A reaches the $80^{\circ} \mathrm{C}$ threshold first at $\tau_{A}^{*}=545 \mathrm{~s}$. Case B absorbs heat longer with $\tau_{B}^{*}=612 \mathrm{~s}$. The optimum configuration has the largest useful operation with $\tau_{\mathrm{opt}}^{*}=645 \mathrm{~s}$.

In the right half of Figure 3, the melt fraction curves for Case A and Case B are nearly identical. The optimum configuration has a slower rate of melting initially, and a higher rate of melting in the latter period of heat addition. The $f^{*}$ values

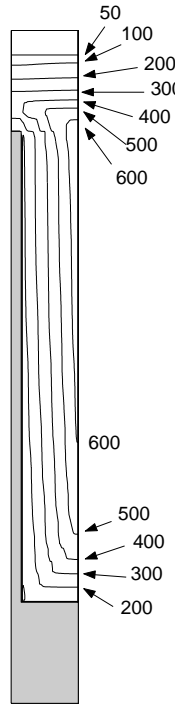

Case A

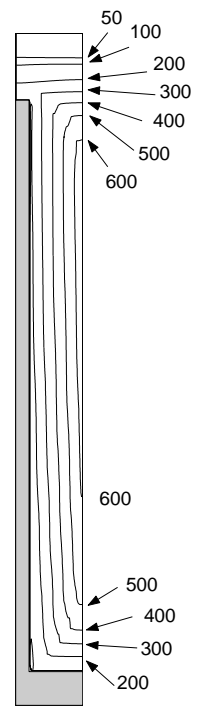

Case B

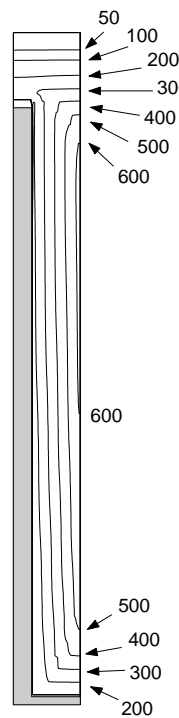

Optimum
Fig. 4. Progression of the melt front for three configurations of the heat sink. Contours of melt front location are labelled with the time in seconds. Parameter values for Case A, Case B, and the optimum solution are defined in Table II. The volume occupied by the aluminum fin is shaded gray.

for Case A, Case B, and the optimum geometry are 0.86, 0.95, and 1.0 , respectively. (Note that it is not easy to read $f^{*}$ values from Figure 3 because $f^{*}$ is the value of $f$ when $t=\tau^{*}$.) The $f^{*}$ values indicate that the optimum geometry makes the most effective use of the PCM, since the PCM is fully melted when the base temperature reaches the threshold temperature.

Figure 4 depicts the progression of the melt front for the three model configurations having the transient response in Figure 3. The numerical values on the right side of the contour plots are the times in seconds for each melt front position. The domain volume occupied by the aluminum fin is shaded gray.

Notice that melting starts at the top of the domain: the first melt contour in each case is at $t=50 \mathrm{~s}$ and is parallel to the top boundary. This explains the initial increase in melt fraction in the right hand side of Figure 3. Although the heat load is imposed at the bottom of the domain, there is also heat gain from the ambient at the top of the domain. The boundary condition at the top allows heat to flow immediately into the PCM. The model does not include the thermal mass of the insulation and case at the top of the fin.

By $t=200 \mathrm{~s}$ a layer of PCM adjacent to the fin has melted. For $t>200 \mathrm{~s}$, the melt front progresses as a nearly vertical plane in most of the space between the fin and the right symmetry boundary. The slight inclination of the melt front (from exactly vertical) corresponds to a small temperature gradient along the length of the fin.

The melt front in the gap between the fin tip and the top boundary moves vertically downward as a nearly horizontal plane. Only in the immediate vicinity of the fin tip does the downward moving melt front interact with the horizontally moving melt front.

The melt front plots in Figure 4 also show the movement of the $T=T_{m}$ isotherm during the simulations. Complete temperature field histories are not shown here to conserve space: 


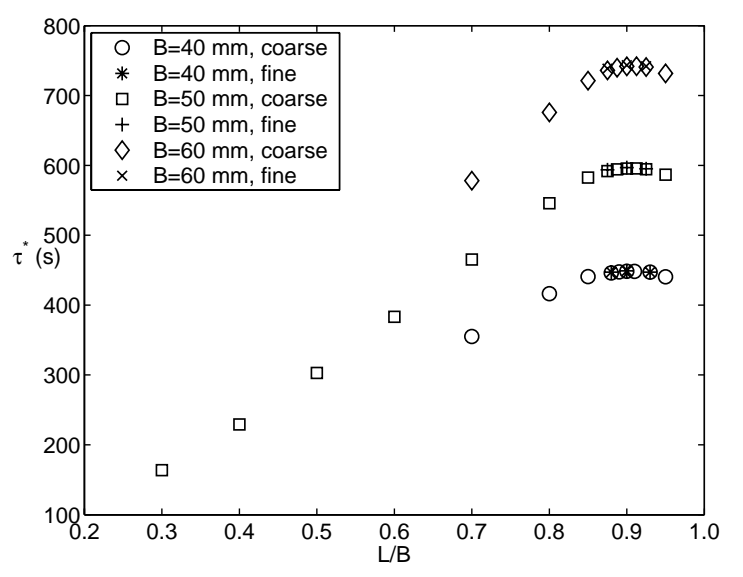

Fig. 5. Variation of $\tau^{*}$ with fin length for $B=40,50$, and $60 \mathrm{~mm} . b / B=$ 0.15 and $s / w=0.15$ are constant. Coarse and Fine refer to the computational mesh used to obtain the results.

each transient would need to be displayed as a sequence of frames. The temperature field plots (if they were shown) would demonstrate that there is very little temperature variation in the aluminum fin compared to the temperature variation within the PCM.

\section{B. Sequential Variation of Geometric Parameters}

Figure 5 shows the effect of varying $L / B$ on $\tau^{*}$. The maximum $\tau^{*}$ occurs at $L / B \approx 0.9$ for $B=40,50$, and $60 \mathrm{~mm}$. The value of $\tau^{*}$ increases linearly with $B$ for these values of $B$. Thus, the heat storage capacity of the assembly is proportional to the overall height of the assembly near the optimum $L / B \approx 0.9$.

After the results in Figure 5 were obtained, we found the value of $s / w$ that maximized $\tau^{*}$ when $L / B$ was held at 0.9 . The results of this optimization phase are shown in Figure 6. The optimal fin half-thickness is $s / w=0.288$ when $L / B=0.9$ and $b / B=0.15$.

Next, with $L / B=0.9$ and $s / w=0.288$, the value of $b / B$ that maximized $\tau^{*}$ was obtained. These results are shown in Figure 7. The optimum appears near $b / B=0$, which makes sense as long as the heat input at the base is uniform. For the fin assembly shown in Figure 1, where the heat input is over a limited part of the fin base, one would expect the optimum base thickness to be somewhat greater than zero. In a heat sink with $b / B \approx 0$ the spreading resistance will significantly reduce the effectiveness of the PCM farthest from the heat source.

\section{Final Optimization}

The sequential optimization process described in the preceding section does not produce the true optimal design. Only one parameter was varied at a time, so interactions of the parameters are neglected. However, the sequential optimization process does lead to significant improvement in performance. After the sequential optimization procedure, we used a trial-and-error approach to adjust the geometrical parameters in search of the true optimum.

We also made slight change to the model for the final optimization. The results described in the preceding section were

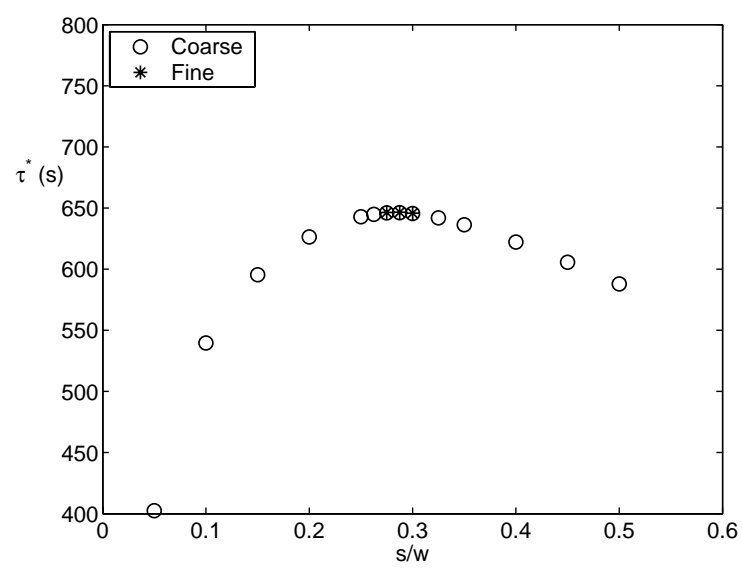

Fig. 6. Variation of $\tau^{*}$ with fin width for a $L / B=0.9, B=50 \mathrm{~mm}$, and $b / B=0.15$. Coarse and Fine refer to the computational mesh used to obtain the results.

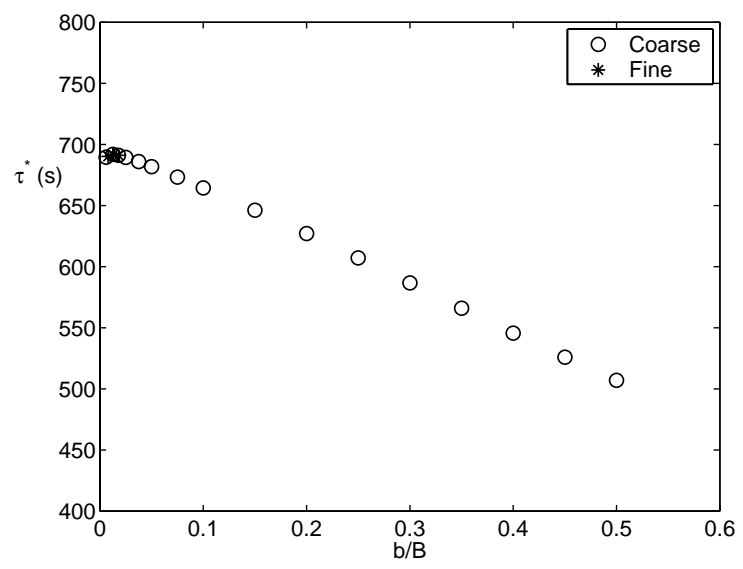

Fig. 7. Variation of $\tau^{*}$ with fin base thickness for a $L / B=0.9, B=50 \mathrm{~mm}$, and $s / w=0.288$. Coarse and Fine refer to the computational mesh used to obtain the results.

obtained with $c_{\ell}=c_{s}=2950 \mathrm{~J} / \mathrm{kg} / \mathrm{K}$. For the final optimization we used $c_{\ell}=2510 \mathrm{~J} / \mathrm{kg} / \mathrm{K}$ and $c_{s}=2950 \mathrm{~J} / \mathrm{kg} / \mathrm{K}$ as indicated in Table I. Because of the smaller heat capacity of the liquid phase, the $\tau^{*}$ values for the final optimization phase are about six percent smaller than the $\tau^{*}$ values for a given set of $L / B$, $b / B$, and $s / w$. The optimal values of $L / B, b / B$, and $s / w$ did not change.

The final, trial-and-error optimization gives $L / B=0.8875$, $s / w=0.275$, and $b / B=0.0125$ as the design with the maximum $\tau^{*}$. Table III summarizes the result of this final analysis, and it shows how each stage of the optimization contributes to an increase in $\tau^{*}$. (The order in which the geometric parameters was varied is arbitrary, of course.)

Table III shows values of $\tau^{*}, f^{*}$, and $\tau_{\infty}$. Our primary objective was to maximize $\tau^{*} . \tau_{\infty}$ is the time during a simulation when the PCM is completely melted. For good designs, $\tau_{\infty} \approx \tau^{*}$ because storing energy in the latent of the PCM is much more effective than storing sensible heat in either the PCM or the aluminum. Also note that $\tau_{\infty}=\tau^{*}$ requires $f^{*}=1$. If $f^{*}=1$, then the PCM has been completely melted when the base reaches its threshold temperature. If $f^{*}<1$ then more PCM is available for absorbing heat, but the temperature 
TABLE III

VALUES OF THE FIGURES OF MERIT AT THE END OF EACH STATE OF THE SEQUENTIAL OPTIMIZATION. VALUES OF $\tau^{*}$ AND $\tau_{\infty}$ ARE IN SECONDS.

\begin{tabular}{lcccccc} 
& \multicolumn{3}{c}{ Best Parameters } & \multicolumn{3}{c}{ Figures of Merit } \\
Vary & $L / B$ & $s / w$ & $b / B$ & $\tau^{*}$ & $f^{*}$ & $\tau_{\infty}$ \\
\hline$L / B$ & 0.900 & 0.150 & 0.150 & 559 & 0.87 & 683 \\
$s / w$ & 0.900 & 0.288 & 0.150 & 606 & 1 & 596 \\
$b / B$ & 0.900 & 0.288 & 0.0125 & 644 & 1 & 634 \\
Final & 0.8875 & 0.275 & 0.0125 & 645 & 1 & 645 \\
\hline
\end{tabular}

gradient inside the heat sink assembly is too large and the base has overheated.

The performance gain obtained at the true optimum is a minor improvement over the results of the sequential optimization. At the final optimum, $\tau^{*}$ is only one second longer than at the results of the sequential optimization.

\section{CONCLUSIONS}

We have optimized the geometry of a repeating module in the heat sink. The optimization does not consider the effects of convection in the molten PCM, edge effects on the ambient boundary, and heat spreading effects in the heat sink base.

For the two-dimensional repeating module, our optimum solution leads to these conclusions.

- There should be a small gap between the fin tip and the top of the enclosure. This gap serves to limit conduction between the ambient and the tip of the fin, without sacrificing too much PCM that is not in contact with the fin.

- The optimum geometry has a thin base $(b / B=0.0125)$ and sufficiently thick fins $(s / w=0.275)$.

- The thickness of the base will not apply to configurations where the heat input is not uniform over the base of the fin assembly.

The numerical model also gives insight into the interaction between heat conduction and phase change. In particular, due to the nearly isothermal metal fin, most of the melt front progresses in the direction normal to the direction of the external heat flux vector.

The two most valuable improvements to the model would be to include multiple fins and heat spreading in the base, and to allow free convection in the melted PCM. Both of these extensions will require optimization of the code to reduce execution time.

For the geometry scales under consideration, the role of convection is believed to be unimportant. Concern about motion of the liquid PCM can be avoided by using a dry PCM, microencapsulation, or embedding the PCM in an expanded metal foam. A serious problem with the use of metal foam in this application is that it would tend to enhance the heat gain from the ambient. Our analysis shows that there is significant melting of the PCM due to ambient heat gain. The optimal solution using the fin design requires a gap between the fin tip and the external case to reduce transport of heat from the ambient into the core of the PCM.

Finally, it is important to remember that the optimization results presented here were obtained for a particular PCM, a fixed fin spacing, a fixed heat load from the electronics, a fixed conductance to the ambient, and a fixed ambient temperature. Finding the optimum fin geometry over the complete range of operating conditions will require further effort.

\section{REFERENCES}

[1] D. Pal and Y.K. Joshi. Transient thermal management of an avionics module using solid-liquid phase change materials (PCMs). In Proceedings of the ASME National Heat Transfer Conference, volume 7 of HTD-Vol.329, pages 145-155. ASME, 1996.

[2] John Patrick O'Conner and Richard M. Weber. Thermal management of electronic packages using solid-to-liquid phase change techniques. International Journal of Microcircuits and Electronic Packaging, 20:593-601, 1997.

[3] Candice A. Bauer and Richard A. Wirtz. Thermal characteristics of a compact, passive thermal energy storage device. In Proceedings of the 2000 ASME International Mechanical Engineering Congress and Exposition. ASME, Nov 2000. Paper No. 2-e-2-1.

[4] Ning Zheng and Richard A. Wirtz. Methodology for designing a hybrid thermal energy storage heat sink. In Proceedings of the 2001 ASME International Mechanical Engineering Congress and Exposition. ASME, Nov 2001. Paper No. 2-16-2-10.

[5] Ning Zheng and Richard A. Wirtz. Figures of merit for hybrid thermal energy storage units. In Proceedings of the 2001 ASME National Heat Transfer Conference. ASME, Jun 2001. Paper No. NHTC2001-20027.

[6] A.J. Fosset, M.T. Maguire, A.A. Kudirka, F.E. Mills, and D.A. Brown. Avionics passive cooling with microencapsulated phase change materials. Journal of Electronic Packaging, 120:238-242, Sep 1998.

[7] Marc Hodes, Randy D. Weinstein, Stephen J. Pence, Jason M. Piccini, Lou Manzione, and Calvin Chen. Transient thermal management of a handset using phase change material (PCM). Journal of Electronic Packaging, 124:419-426, Dec 2002.

[8] Patrick Brousseau and Marcel Lacroix. Numerical simulation of a compact multi-layer phase change material energy storage system. In Fundamentals of Phase Change: Sublimation and Solidification, HTD-Vol. 286, pages 13-20. ASME, 1994.

[9] A. Haji-Sheikh, J. Eftekhar, and D. Lou. Properties of paraffin wax as a thermal storage medium. In Proceedings of the AIAA/ASME Joint Thermophysics, Fluids, Plasma and Heat Transfer Conference. AIAA, June 1982.

[10] Benjamin Gebhart, Yogesh Jaluria, Roop L. Mahajan, and Bahgat Sammakia. Buoyancy-Induced Flows and Transport. Hemisphere, New York, 1988.

[11] V. R. Voller and C.R. Swaminathan. General source-based method for solidification phase change. Numerical Heat Transfer, Part B, 19:175$189,1991$.

[12] Jason McKenzie Leland. Numerical optimization of a phase change heat sink. MSME Thesis, Portland State University, August 2002.

[13] E.R.G. Eckert and Jr. Drake, Robert M. Analysis of Heat and Mass Transfer. McGraw-Hill, New York, 1972. 